\title{
Hepatic capillariasis in rats: a new model for testing antifibrotic drugs
}

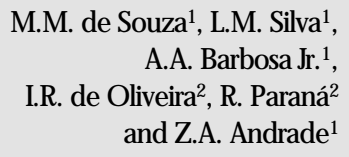

M.M. de Souza ${ }^{1}$, L.M. Silva ${ }^{1}$, A.A. Barbosa Jr. ${ }^{1}$, I.R. de Oliveira², R. Paraná2 and Z.A. Andrade ${ }^{1}$

\author{
${ }^{1}$ Centro de Pesquisas Gonçalo M oniz, Fundação O swaldo Cruz, and \\ ${ }^{2}$ Faculdade de Medicina, Universidade Federal da Bahia, Salvador, BA, Brasil
}

\section{Correspondence}

Z.A. Andrade

Centro de Pesquisas Gonçalo Moniz FIOCRUZ

Rua Valdemar Falcão, 121

40295-001 Salvador, BA

Brasil

Fax: + 55-71-356-4292

E-mail: zilton@ cpqgm.fiocruz.br

Received April 5, 2000

Accepted August 2, 2000

\section{Abstract}

Rats infected with the helminth Capillaria hepatica regularly develop septal hepatic fibrosis that may progress to cirrhosis in a relatively short time. Because of such characteristics, this experimental model was selected for testing drugs exhibiting antifibrosis potential, such as pentoxifylline, gadolinium chloride and vitamin A. Hepatic fibrosis was qualitatively and quantitatively evaluated in liver samples obtained by partial hepatectomy and at autopsy. The material was submitted to histological, biochemical and morphometric methods. A statistically significant reduction of fibrosis was obtained with pentoxifylline when administered intraperitoneally rather than intravenously. Gadolinium chloride showed moderate activity when administered prophylactically (before fibrosis had started), but showed a poor effect when fibrosis was well advanced. No modification of fibrosis was seen after vitamin A administration. Hydroxyproline content was correlated with morphometric measurements. The model appears to be adequate, since few animals die of the infection, fibrosis develops regularly in all animals, and the effects of different antifibrotic drugs and administration protocols can be easily detected.

\section{Key words}

- Capillaria hepatica

- Hepatic fibrosis

- Septal fibrosis

- Antifibrotic drugs

\section{Introduction}

Several animal models of hepatic fibrosis have been utilized for testing drugs exhibiting antifibrotic potential. The models of hepatic fibrosis utilized are mainly those obtained in rats by repeated injections of carbon tetrachloride, by injections of porcine serum or following total main bile duct obstruction (1). Mice infected with Schistosoma mansoni have also been used (2). However, all of these models present limitations, especially due to individual variation in fi- brosis development and high mortality, not counting the prolonged time involved and the high cost of some of them. Recently, it has been shown that rats chronically infected with the helminth Capillaria hepatica regularly develop progressive and diffuse septal hepatic fibrosis (3) similar to that seen in rats repeatedly injected with whole porcine serum $(4,5)$. Fibrosis starts around the 40th day after infection, when the worms are already dead and the parasite-dependent, focal necrotic and inflammatory lesions are undergoing fibrous encapsulation and resorption. 
Because septal fibrosis develops within a relatively short time (40-45 days) in $100 \%$ of the infected rats and shows a progressive course toward cirrhosis, this model has been considered to be one of the most adequate for testing antifibrotic drugs both in terms of their therapeutic and prophylactic effects.

This report concerns the response of hepatic fibrosis associated with $C$. hepatica infection to several drugs exhibiting antifibrotic potential. It was assumed that the results obtained would be worthwhile both for testing the model and the drugs.

\section{Material and Methods}

Wistar rats of both sexes weighing 170 to $300 \mathrm{~g}$ were infected with approximately 1,000 embryonated eggs of $C$. hepatica each, administered by gavage. The eggs were obtained from the livers of experimentally infected rats. The liver tissue was homogenized in a blender at 1,000 rpm, washed repeatedly in tap water, and left to sediment, until the supernatant fluid was completely clear. The sediment containing the immature eggs was placed on a Petri dish and covered with gauze humidified with $0.5 \%$ formalin. After 28-30 days at room temperature (26$28^{\circ} \mathrm{C}$ ), the eggs became embryonated and were recovered and used to infect the animals. After inoculation the animals were divided at random into 5 experimental groups of five animals each as follows:

Group I. Infected control. The animals received two weekly subcutaneous $(s c)$ injections of $0.85 \%$ saline.

Groups II and III. The animals were treated daily with $6 \mathrm{mg}$ of pentoxifylline (Trental; Hoechst, São Paulo, SP, Brazil), administered intraperitoneally (ip) and intravenously (iv), respectively.

Group $I V$. The animals were treated with $1 \mathrm{ml}$ of a $4 \mathrm{mM}$ solution of gadolinium chloride (Sigma-66H3405; Sigma Chemical Co., St. Louis, MO, USA) administered $i v$.

Group $V$. The animals were treated with vitamin A (Arovit; Roche, São Paulo, SP, Brazil) twice a week by the $s c$ route at the dose of 50,000 IU up to a total of 200,000 IU.

Treatment was started for all the groups on the 25th day after inoculation. To follow the course of treatment and to evaluate the preventive potential of the drugs, the animals were submitted to partial hepatectomy (surgical liver biopsy) one week after the end of treatment (45th day of infection). Treatment was resumed one week later, up to the time of sacrifice on the 95th day following infection. Table 1 summarizes the experimental protocols used.

For histological study, fragments of the liver collected during all the experimental phases were immediately fixed in Bouin's fixative for $6 \mathrm{~h}$, preserved in $70 \%$ alcohol and routinely embedded in paraffin. Sections were stained with hematoxylin and eosin. In addition, the sirius red method for collagen was also used (6). The slides were blindly and independently evaluated by two pathologists

Table 1 - Schedule of treatment of fibrosis in rats with septal hepatic fibrosis associated with Capillaria hepatica infection.

*Time considered from the day of inoculation.

\begin{tabular}{llcccc}
\hline Group & Dose & Route & $\begin{array}{c}\text { Duration of } \\
\text { treatment (days)* }\end{array}$ & Hepatectomy* & Sacrifice* \\
\hline I - Saline & $1 \mathrm{ml}$ & sc & $25-45$ & 45th and 65th & 95th \\
II - Pentoxifylline & $6 \mathrm{mg}$ & ip & $25-45$ & 45 th & 95 th \\
III - Pentoxifylline & $6 \mathrm{mg}$ & iv & $25-45$ & 45 th & 95 th \\
IV - Gadolinium chloride & $4 \mathrm{mM}$ & iv & $25-65$ & 45th & 95 th \\
V - Vitamin A & 50,000 IU & sc & $25-39$ & 44th & 108th
\end{tabular}


by a semi-quantitative method, which considered fibrosis as absent $(0)$, mild $(+)$, moderate $(++)$ and marked $(+++)$ (see Figure 1). In case of disagreement, a consensus was reached before decoding the slides. Other portions of the liver were separated and submitted to the method of Bergman and Loxley (7) for measurement of hydroxyproline content.

Morphometric measurements were made on histological slides stained by the sirius red method and submitted to a computer image analyzing system (Leica Quantimet Q500MC, Cambridge, England). A total sectional area of $17.01 \times 10^{6} \mu \mathrm{m}^{2}$ per case was evaluated. A Leica Microstar IV microscope with a $4 \mathrm{X}$ objective was used to examine 9 microscopic fields at random. The sectional area of the fibrous tissue, red stained, was directly measured and calculated as percent of the total area examined.

An extra group was used to test the effect of gadolinium chloride on Kupffer cells. Normal rats were injected with a single dose or with 3 repeated doses of gadolinium chloride ( $4 \mathrm{mM} /$ animal) one every $72 \mathrm{~h}$. Twentyfour hours after the last injection, the animals received $1 \mathrm{ml}$ of $10 \%$ India ink (colloidal carbon) $i v$.

Normal rats injected with India ink served as controls. The animals were sacrificed 24$48 \mathrm{~h}$ after injection of India ink and their livers submitted to histological examination by means of paraffin sections lightly stained with hematoxylin.

\section{Results}

Scattered focal lesions containing disintegrating parasites and their eggs varied in quantity but septal fibrosis was diffuse and appeared regularly in all infected untreated animals. By the 107th day of infection, the focal lesions had become small, encapsulated and frequently calcified, while septal fibrosis was more marked, being sometimes associated with areas of nodular regeneration of the hepatic parenchyma (Figure 1A).
Evaluation of the extent of septal fibrosis by a semi-quantitative histological method demonstrated that the groups treated $i p$ with pentoxifylline exhibited a considerable decrease or even total absence of septal fibrosis (Figure 1B, C and D) as compared to the
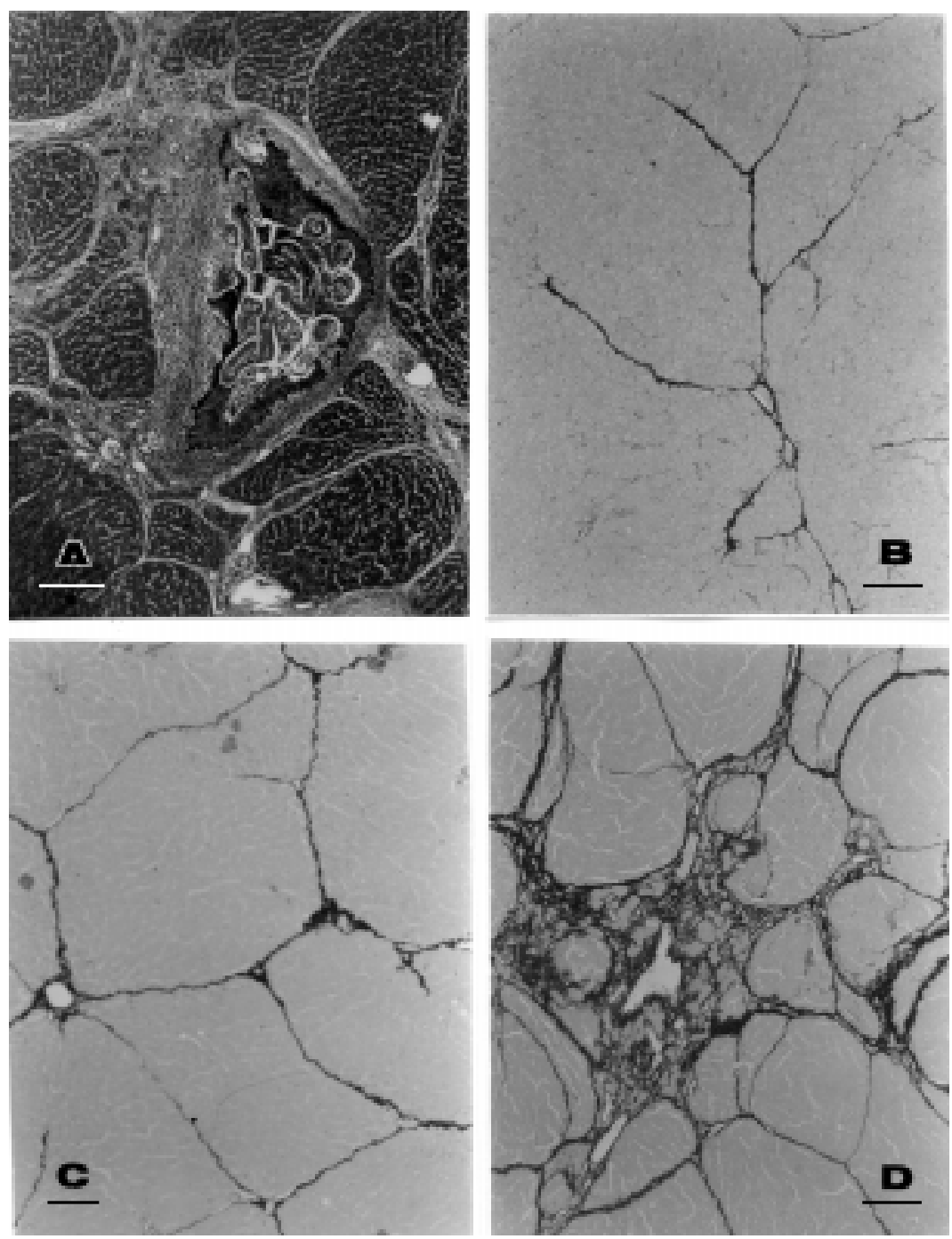

Figure 1 - A, Disintegrating adult worms (Capillaria hepatica) are seen at the center of the picture, surrounded by a necrotic-inflammatory reaction and a fibrous capsule. Septal fibrosis and regenerating hepatic nodules are also present. $H \& E, 120 X$ (bar represents 100 $\mu \mathrm{m})$. B, Representative of the mild (+) degree of septal fibrosis observed in rats treated with pentoxifylline by ip administration. Septa are few, thin and incomplete, and the majority terminate abruptly within the hepatic parenchyma. Sirius red staining, 100X (bar represents $100 \mu \mathrm{m}) . \mathrm{C}$ and D, Representatives of the moderate (++) and marked (+++) degrees of septal fibrosis observed in infected rats treated with antifibrotic drugs. C, Septa circumscribe irregular areas of the hepatic parenchyma and show points of increased thickness (treated with gadolinium chloride); D, marked septal fibrosis delimiting hepatic nodules (cirrhosis) and forming areas of condensation, treated with vitamin A. Sirius red staining, 100X (bars represent $100 \mu \mathrm{m}$ ). 
control group which presented a marked degree of fibrosis.

Morphometric evaluation confirmed the results obtained by semi-quantitative histology. The mean values for representative sectional areas of the liver revealed a considerable reduction of fibrosis for the groups treated with pentoxifylline (given $i p$ ), in comparison to untreated controls $(\mathrm{P}<0.005)$ (Table 2). The administration of vitamin A failed to modify the degree of liver fibrosis in a significant manner.

Hydroxyproline concentration (Table 3) also agreed with the data mentioned above but, in addition to indicating a statistically significant decrease in fibrosis for the group treated with pentoxifylline, it also showed similar results for gadolinium when administered before fibrosis had become established (prophylactic treatment).

The uptake of colloidal carbon by sinusoidal cells in normal rats treated with gadolinium chloride failed to show any differ-

Table 2 - Comparison of the mean values obtained by morphometric measurement of fibrosis in liver sections stained with sirius red from the various experimental groups.

$* \mathrm{P}<0.05$ compared to control (Group I) (Student-Newman-Keuls' test). $\mathrm{N}=5$ for all groups.

\begin{tabular}{lcl}
\hline Groups & Mean \pm SD $\left(\mu \mathrm{m}^{2}\right)$ & \multicolumn{1}{c}{ Treatment } \\
\hline I & $19.28 \pm 2.17 \times 10^{4}$ & PBS $(0.85 \%)$ \\
II & $6.42 \pm 6.65 \times 10^{4 *}$ & Pentoxifylline (ip) \\
III & $16.94 \pm 6.76 \times 10^{4}$ & Pentoxifylline (iv) \\
IV & $14.68 \pm 2.69 \times 10^{4}$ & Gadolinium chloride (preventive) \\
V & $12.50 \pm 3.87 \times 10^{4}$ & Vitamin A
\end{tabular}

Table 3 - Comparison of the mean values obtained by measurement of hepatic hydroxyproline content in the various experimental groups.

$* \mathrm{P}<0.05$ compared to control (Group I) (Student-Newman-Keuls' test). $\mathrm{N}=5$ for all groups.

\begin{tabular}{lll}
\hline Groups & Mean \pm SD $\left(\mu \mathrm{m}^{2}\right)$ & \multicolumn{1}{c}{ Treatment } \\
\hline I & $8.84 \pm 1.91$ & PBS (0.85\%) \\
II & $5.69 \pm 0.49 *$ & Pentoxifylline (ip) \\
III & $7.85 \pm 1.54$ & Pentoxifylline (iv) \\
IV & $6.41 \pm 0.89 *$ & Gadolinium chloride (preventive) \\
V & $7.08 \pm 0.97$ & Vitamin A
\end{tabular}

ence when compared to untreated controls, with a similar number of sinusoidal cells containing carbon particles observed in both cases.

Data were analyzed by one-way ANOVA and the Student-Newman-Keuls test for group comparison.

\section{Discussion}

The model of septal fibrosis developed in rats infected with $C$. hepatica appeared to be adequate for testing antifibrotic drugs. Hepatic fibrosis was constant, uniform and progressive in infected controls and was significantly, albeit variably, affected by antifibrotic treatment. Since septal fibrosis does not appear before the 30th day of infection, drugs can be reliably tested with the present model for their prophylactic value when administered around the 25th day of infection or for their therapeutic action when administered after the 50th day.

Clear-cut positive results were obtained with pentoxifylline treatment, with the route of drug administration appearing to be crucial. The ip route yielded better results than the $i v$ route. It is rather difficult to explain this finding, since the action of pentoxifylline seems to be quite complex. Pentoxifylline (methylxanthine) is known to inhibit collagen synthesis, suppress Kupffer cell activation and promote decrease of serum TNF$\alpha(8,9)$. It can also inhibit collagen deposition and connective tissue cell proliferation (10). Probably, the plasma concentration and half-life of the drug differ according to the route of administration. Also, when injected $i p$, the drug would reach sinusoidal cells in the liver more rapidly or at more adequate concentrations than when systemically administered.

Sinusoidal cells play a pivotal role in septal fibrosis (11). As a matter of fact, the possibility of the drug interfering with sinusoidal cells stimulated us to use gadolinium chloride in this investigation. Gadolinium is 
claimed to block phagocytosis by Kupffer cells (12-15).

In the present investigation, gadolinium initially yielded clear-cut antifibrotic results, but did not avoid the progress of fibrosis thereafter. The drug causes phenotypic alteration in Kupffer cells, causing them to produce excess TNF- $\alpha$ (14). Surprisingly, our results with the India ink test did not indicate that gadolinium altered the phagocytic capacity of Kupffer cells.

Vitamin A has yielded controversial antifibrotic results. High doses are considered to induce fibrosis in some studies (16), while others have found the opposite (17). High doses of vitamin A cause the Ito cells to be loaded with fat droplets. This may even depress the synthetic machinery of the cell through a space-occupying effect. Our results failed to show an antifibrotic effect when high doses of vitamin A were administered to rats with $C$. hepatica-associated septal fibrosis of the liver.

Further studies with different doses and protocols, with the present drugs or other compounds, should better characterize the antifibrotic properties of the agents used. The effects observed can also be compared in different models, since the antifibrotic activity may vary according to the pathogenesis of hepatic fibrosis. Hepatic fibrosis is usually associated with chronic inflammation and/or hepatocellular necrosis. Septal fibrosis of the liver induced in rats either by porcine serum $(2,5)$ or by $C$. hepatica infection (3) is not preceded by outstanding necrosis or inflammation. Septal fibrosis seems to have an immunological basis (18), which turns the present model still more interesting. In conclusion, the results of the present investigation indicate that the model of hepatic septal fibrosis associated with $C$. hepatica infection of the rat is adequate for studies concerning the response of fibrosis to drug treatment and that it may also be valuable for investigations concerning the pathogenesis of liver fibrosis.

\section{References}

1. Tsukamoto $\mathrm{H}$, Matsuoka $\mathrm{M} \&$ French $\mathrm{SW}$ (1990). Experimental models of hepatic fibrosis: A review. Seminars in Liver Disease, 10: 56-65.

2. Andrade ZA \& Grimaud J A (1986). Evolution of the schistosomal hepatic lesions in mice after curative chemotherapy. American J ournal of Pathology, 124: 59-65.

3. Ferreira LA \& Andrade ZA (1993). Capillaria hepatica: a cause of septal fibrosis of the liver. Memórias do Instituto Oswaldo Cruz, 88: 441-447.

4. Andrade ZA (1991). Contribution to the study of septal fibrosis of the liver. International J ournal of Experimental Pathology, 72: 553-562.

5. Paronetto F \& Popper H (1966). Chronic liver injury induced by immunologic reactions. Cirrhosis following immunization with heterologous sera. American J ournal of Pathology, 40: 1087-1101.

6. J unqueira LCU, Bignolas $G \&$ Brentani $R$ (1979). Picrosirius staining plus polarization microscopy, a specific method for collagen detection in tissue sections. His- tochemical J ournal, 11: 447-455.

7. Bergman I \& Loxley R (1963). Improved and simplified methods for the spectrophotometric determination of hydroxyproline. Analytical Chemistry, 35: 1961-1965.

8. Duncan MR, Hasan A \& Berman B (1995). Pentoxifylline, pentifylline, and interferons decrease type I and III procollagen mRNA levels in dermal fibroblasts: evidence for mediation by nuclear factor 1 down-regulation. J ournal of Investigative Dermatology, 104: 282-286.

9. Kozaki K, Egawa H, Bermudez L, Keefe EB, So SK \& Esquivel CO (1995). Effects of pentoxifylline pretreatment on Kupffer cells in rat liver transplantation. Hepatology, 21: 1079-1082.

10. Romanelli RG, Caligiuri A, Carloni V, DeFranco R, Montalto P, Ceni E, Casini A, Gentilini P \& Pinzani M (1997). Effect of pentoxifylline on the degradation of procollagen type I produced by human hepatic stellate cells in response to transforming growth factor-beta 1 . British J ournal of Pharmacology, 122: 1047-1054.
11. Carloni V, Romanelli RG, Pinzani M, Laffi $G \&$ Gentilini $P$ (1996). Expression and function of integrin receptors for collagen and laminin in cultured human hepatic stellate cells. Gastroenterology, 110: 1127-1136.

12. Kamei T, Callery MP \& Flye MW (1990). Kupffer cell blockade prevents induction of portal venous tolerance in rat cardiac allograft transplantation. J ournal of Surgical Research, 48: 393-396.

13. Vidal C, Gonzalez-Quintela A \& CuervasMons V (1993). Influence of Kupffer cell phagocytosis blockade on the production of ovalbumin-specific IgE and IgG1 antibodies in an experimental model. Clinical and Experimental Allergy, 23: 15-20.

14. Aril S, Monden $K$, Adachi $Y$, Zhang $W$, Higashitsuji $H$, Furutani M, Fujita S, Nakamura T \& Imamura M (1994). Pathogenic role of Kupffer cell activation in the reperfusion injury of cold-preserved liver. Transplantation, 58: 1072-1077.

15. Rai RM, Zhang J X, Clemens MG \& Diehl AM (1996). Gadolinium chloride alters the 
acinar distribution of phagocytosis and balance between pro- and anti-inflammatory cytokines. Shock, 6: 243-247.

16. Bioulac-Sage $P$, Quinton $A$, Saric J, Grimaud J Á, Mourey MS \& Balabaud C (1998). Chance discovery of hepatic fibro- sis in patient with asymptomatic hypervitaminosis A. Archives of Pathology and Laboratory Medicine, 112: 505-509.

17. Yamane $M$, Tanaka $Y, M$ arumo $F \&$ Sato $C$ (1993). Role of hepatic vitamin A and lipocyte distribution in experimental hepatic fibrosis. Liver, 13: 282-287.

18. Bhunchet E, Eishi Y \& Wake K (1996). Contribution of immune response to the hepatic fibrosis induced by porcine serum. Hepatology, 23: 811-817. 